\title{
Fitoterapia para o tratamento da diabetes: um estudo do potencial hipoglicemiante de plantas
}

\author{
Phytotherapy for the treatment of diabetes: a study of the hypoglycemiant potential of plants \\ Fitoterapia para el tratamiento de la diabetes: un estudio del potencial hipoglucémico de \\ las plantas
}

Thallysson Jose Dourado de Sousa ${ }^{1 *}$, Adayran Raposo Lacerda ${ }^{1}$, Camila Pinheiro Santiago Silva ${ }^{1}$, Monick Nielly Miranda Pinto ${ }^{1}$, Simão Pedro Xavier Rocha Martins ${ }^{1}$, Tatielle Gomes Dias ${ }^{1}$, Kassyo Lenno Sousa Dantas ${ }^{1}$, Milena Sousa Freitas ${ }^{1}$, Domingos Magno Santos Pereira ${ }^{1}$, Cristiane Santos Silva e Silva Figueiredo'.

\section{RESUMO}

Objetivo: Discorrer a atividade antidiabética e as suas principais formas de ação em meio ao distúrbio metabólico de insulina presentes na circulação sanguínea por plantas encontradas na biodiversidade vegetal do Brasil. Revisão bibliográfica: Quatro plantas empregadas como agentes medicinais foram escolhidas a fim de que a sua possível atuação através de extratos sobre o distúrbio de insulina presentes na circulação sanguínea, já reconhecida por alguns agrupamentos humanos, fosse identificado, as quais são: Bauhinia forficata, Pterocarpus marsupium roxburgh, Morus nigra L e Momordica charantia L. O estudo deu-se mediante a compilação de dados, os quais ponderam efeitos antidiabéticos contidos em cada uma das plantas descritas ao longo da pesquisa e os seus mecanismos de desempenho em relação à fisiologia do controle glicêmico no organismo, através de estudos pré-clínicos em sua maioria. Considerações finais: Foi possível demonstrar os efeitos positivos da fitoterapia através de cada uma das plantas explanadas ao longo da pesquisa no tratamento de diabetes, seja por seu efeito direto ou como coadjuvante durante a terapia.

Palavras-chave: Fitoterapia, Plantas medicinais, Diabetes, Agente antidiabético.

\begin{abstract}
Objective: To discuss the antidiabetic activity and its main forms of action in the midst of metabolic disturbance of insulin present in the bloodstream by plants found in plant biodiversity in Brazil. Bibliographic review: Four plants used as medicinal agents were chosen so that their possible action through extracts on the insulin disorder present in the bloodstream, already recognized by some human groups, was identified, which are: Bauhinia forficata, Pterocarpus marsupium roxburgh, Morus nigra $L$ and Momordica charantia $L$. The study was carried out through the compilation of data, which weighed antidiabetic effects contained in each of the plants described throughout the research and their performance mechanisms in relation to the physiology of control glycemic in the body, through pre-clinical studies mostly. Final considerations: It was possible to visualize the positive effects of herbal medicine through each of the plants explained throughout the research in the treatment of diabetes, either for its direct effect or as an adjunct during therapy.
\end{abstract}

Keywords: Phytotherapy, Medicinal plants, Diabetes, Antidiabetic agent.

\section{RESUMEN}

Objetivo: Discutir la actividad antidiabética y sus principales formas de acción en medio de la alteración metabólica de la insulina presente en el torrente sanguíneo por plantas que se encuentran en la biodiversidad

${ }^{1}$ Universidade CEUMA (UNICEUMA), Imperatriz - MA. *E-mail: br526thallyssondourado@gmail.com 
vegetal en Brasil. Revisión bibliográfica: Se eligieron cuatro plantas utilizadas como agentes medicinales para que se identificara su posible acción a través de extractos sobre el trastorno de la insulina presente en el torrente sanguíneo, ya reconocido por algunos grupos humanos, que son: Bauhinia forficata, Pterocarpus marsupium roxburgh, Morus nigra L. y Momordica charantia L. El estudio se llevó a cabo mediante la recopilación de datos, en los que se ponderaron los efectos antidiabéticos contenidos en cada una de las plantas descritas a lo largo de la investigación y sus mecanismos de actuación en relación con la fisiología del control glucémico en el organismo, a través de métodos preclínicos. estudios en su mayoría. Consideraciones finales: Se pudo visualizar los efectos positivos de la fitoterapia a través de cada una de las plantas explicadas a lo largo de la investigación en el tratamiento de la diabetes, ya sea por su efecto directo o como coadyuvante durante la terapia.

Palabras clave: Fitoterapia, Plantas medicinales, Diabetes, Agente antidiabético.

\section{INTRODUÇÃO}

Diabetes é uma patologia decorrente do metabolismo que leva a insuficiência ou atuação irregular da insulina, hormônio responsável pela regulação da glicose no sangue que garante energia para o organismo, podendo levar a um quadro de hiperglicemia. As diabetes são classificadas em diabetes mellitus tipo 1 (DM1), tipo 2 (DM2), diabetes gestacional, secundária e diabetes do jovem com início na maturidade, sendo as do tipo 1 e tipo 2 mais recorrente. O diabetes tipo 1 resulta de uma falha autoimune que atinge as células $\beta$ pancreáticas com fatores desencadeantes, ainda sem total compreensão (MARSHALL WJ, et al., 2016).

A DM2 é definida como um distúrbio na produção da insulina, caracterizando uma disfunção no processo de regulação da glicose. A diabetes tipo 2 se desenvolve quando as células $\beta$ inclusas nas ilhotas pancreáticas não conseguem secretar insulina suficiente para atender à necessidade que o organismo precisa para controle do nível de glicose no sangue, iniciando um quadro de aumento da resistência à insulina. Uma minoria de pessoas diagnosticadas com DM2 também tem evidências de autoimunidade às ilhotas (NOVAES VLSS, et al., 2014).

O desencadeamento da resistência à insulina se desenvolve com a deposição de gordura ectópica no fígado e nos músculos. A gordura também pode se acumular no pâncreas e contribuir para o declínio da função das células $\beta$, inflamação das ilhotas e eventual morte das células $\beta$. Atualmente, considera-se ainda desconhecido o que pode ocasionar o DM2, sabe-se que a hiperglicemia é resultante da produção excessiva de glicose e, em menor grau acaba causando a falência do músculo esquelético na absorção da glicose, sendo posteriormente estocado como glicogênio. O DM 2 pode ser descrito das seguintes formas: deficiência das células $\beta$, resistência à insulina e alterações da disponibilidade de glicose mediada por outros fatores que não a insulina (MARSHALL WJ, et al., 2016).

O DM2 é a forma mais comum de diabetes mellitus (DM) no mundo. Geralmente é considerado um diagnóstico de exclusão, ou seja, pacientes são diagnosticados com diabetes tipo 2 porque não apresentam as características típicas do tipo 1. O DM2 provavelmente não é uma condição única. Em todos os pacientes com a doença, existe tanto a resistência à insulina quanto a deficiência relativa de insulina. O diabetes, reflete um grande risco por ser favorável para evolução de outras patologias como doenças cardiovasculares, nefropatia, retinopatia, neuropatia, dentre outras (JACKSON T, et al., 2019).

Segundo a International Diabetes Federation (2019), a média global é que aproximadamente 463 milhões de adultos entre 20 e 79 anos são acometidos pela doença, sendo que $79 \%$ são pessoas residentes em países de baixa e média renda. A diabetes ocasionou 4,2 milhões de mortes no mundo, impactando um gasto na saúde de US $\$ 760$ bilhões em 2019. Já no Brasil, essa patologia acomete mais de 12 milhões de pessoas, sendo mais presente em mulheres, correspondendo a 5,4 milhões e nos homens correspondendo a 3,6 milhões, sendo a DM 2 com maior incidência dos casos. Nos EUA, estima-se que $95 \%$ dos quase 30 milhões de pessoas que vivem com DM2. Outros 86 milhões têm pré-diabetes, colocando-os em alto risco de desenvolver DM2. 
Mesmo diante da complexidade fisiopatológica da DM2, uma vida saudável mediante a realização de práticas físicas rotineiramente, nem sempre se dar a redução de atividade hiperglicêmica e em muitos casos é necessário a utilização de fármacos como alternativa terapêutica (GOMES HG, et al., 2018). Atualmente para o controle glicêmico e metabólico do DM2 há uma série de recursos terapêuticos como modificação da dieta, antidiabéticos orais, insulina e cirurgia metabólica (COSTA AF, et al., 2017).

Portanto tem se analisado que a DM2 corrobora, não somente ao âmbito profissional, como também negativamente para a qualidade de vida, no momento em que os avanços da doença se estadia a estágios que são cruciais para interferência dessa qualidade, mediada pela indisposição, declínio na funcionalidade das atividades vitais, disposição a ansiedade e depressão o que influencia o paciente a coagir a integralização ao meio social, caracterizando-se como uma interferência na sua comodidade social (FARIA HTG, et al., 2013).

Diante a esse cenário e ao índice de progressão a DM tipo 2, segundo o Ministério da Saúde (MS) no Brasil entre os anos de 2006 e 2016, foi constatado um aumento de $60 \%$ em casos diagnosticados, incidindo em um custo próximo a $\mathrm{R} \$ 100$ bilhões por ano, o que se estimava que em 2030 esse custo possa chegar a $\mathrm{R} \$ 406$ bilhões, tornando-se um cenário bem crítico para economia na saúde pública. Nesse contexto destacamos que de acordo ao MS, há uma grande relevância quanto ao preço da insulina, devido ao seu processo tecnológico de fabricação que envolve a manipulação de organismo vivo e por ser um método de difícil cópia o que torna relativamente cara, assim como os demais medicamentos utilizados, contabilizando a um custo entre $R \$ 500$ a $R \$ 800$ a depender do tipo de tratamento utilizado (OLIVEIRA JEP, et al., 2018).

Devido às plantas medicinais possuírem baixo custo e acessibilidade, a sua utilização torna-se uma atividade terapêutica visto que a sua utilização vem se destacado em várias literaturas pela sua ação terapêutica adjacente a DM2 trazendo resultados eficazes quanto aos sintomas e consequências. Algumas espécies vêm sendo avaliadas farmacologicamente e tem demonstrado resultados com potencial antidiabético, algumas com atividade hipoglicêmica, por apresentar compostos análogos aos fármacos usados no tratamento alopático das classes das sulfoniluréias, com efeito estimulador da produção de insulina pelas células $\beta$-pancreáticas (DEFANI MA e OLIVEIRA LEN, 2017).

Sendo assim, este artigo objetiva trazer um compilado de publicações que demonstram cientificamente a eficácia de algumas espécies de plantas, tais como, Bauhinia forficata, Pterocarpus marsupium roxburgh, Morus nigra L, Momordica charantia $L$, para tratamento coadjuvante e/ou alternativo para o diabetes.

\section{REVISÃO BIBLIOGRÁFICA}

A fitoterapia vem sendo atrelada na cultura da humanidade por longas datas, tendo suas maiores evidências e abrangências nas últimas décadas. Baseadas por estudos científicos, como por exemplo o estudo da farmacognosia, uma variedade de plantas medicinais tem apresentado evidências de ações a partir do conhecimento de suas estruturas e composição. Em vista disso, a pesquisa científica moderna vem concretizando estudos para determinar a segurança e eficácia quanto ao uso das plantas medicinais nos tratamentos de diversas patologias, dentre elas a diabetes (MACEDO WDLR, 2019).

Diante da necessidade de novos estudos para determinar compostos e propriedades na constituição de plantas, a biotecnologia tem contribuído fortemente para identificar e analisar alternativas terapêuticas por meio de metabólitos presentes nas plantas, que possuem papel fundamental ao desempenhar diversas interações de defesa contra agentes patogênicos e nocivos (MARSHALL WJ, et al., 2016).

Com isso, pesquisadores buscam alternativas com a realizações de estudos e experimentos que visam apresentar substâncias cruciais para a produção de fármacos promissores em controle de diversas alterações metabólicas e patologias presentes no organismo. O preparo desses medicamentos, se dá com técnicas e estudos de análises de toda a composição de uma planta, observando suas propriedades e mecanismos de ações que podem originar atividades como anti-inflamatórias em combate a inflamação de tecidos, atividade antioxidante, inibindo os efeitos da oxidação e especialmente atividades antidiabéticas, diminuindo a quantidade de glicose presente no sangue, ou seja, colaborando no tratamento de diabetes, além de outras atividades (JACKSON T, et al., 2019). 
Devido a disfunções no funcionamento metabólico de indivíduos portadores de diabetes, novas possibilidades terapêuticas têm surgido para auxiliar no tratamento da patologia. Assim sendo, tem-se as plantas medicinais como uma ferramenta a ser utilizada devido ao fácil acesso e baixo custo. As plantas Bauhinia forficata, Pterocarpus marsupium, Morus nigra $L$ e Momordica charantia $L$. são compostas por substâncias que podem apresentar atividades antidiabéticas, assim regularizando o aumento ou a baixa produção de glicose no organismo que estão associados a fatores patogenéticos que se correlacionam com alterações imunológicas, predisposições genéticas etc. (OLIVEIRA TNFL, et al., 2018). Portanto, a seguir serão discutidas as possíveis ações das plantas já citadas a fim de se obter conhecimento sobre o real potencial antidiabético.

\section{Bauhinia forficata}

A Bauhinia forficata (BF), também conhecida comumente como "pata-de-vaca", pertence à família Fabaceae, que chega a medir aproximadamente 7 a 10 metros de altura, apresentando folhas em formato de pegada de vaca e diversas flores grandes e exóticas, é nativa da Índia, adaptando-se na região da Mata Atlântica. É amplamente utilizada na medicina popular brasileira em forma de chás e infusões para o tratamento da DM, devido sua composição química, que apresenta compostos como saponinas, taninos, terpenóides, e flavonóides como a rutina e quercetina, substâncias responsáveis para desenvolver efeitos farmacológicos no tratamento da diabetes (DEFANI MA e OLIVEIRA LEN, 2017).

Um estudo realizado por Tonelli CA (2019), buscou avaliar a ação do extrato de $B$. forficata em pacientes com diabetes tipo 2. O grupo foi tratado com a ingestão do extrato de $B$. forficata e após 4 meses de tratamento foi analisado os níveis de hemoglobina glicada, níveis de glicose, colesterol, triglicerídeos e entre outros. Os resultados demonstraram a redução significativa de níveis de insulina e hemoglobina glicada após o início do tratamento com o extrato. A partir do exposto entende-se que a utilização do extrato de $B$. forficata é uma alternativa terapêutica promissora para auxiliar no tratamento do diabetes do tipo 2 , com reduções dos índices de glicemia.

Macedo WDLR, et al. (2019), realizou um estudo entrevistando idosos com DM tipo 2 em uma unidade básica de saúde, o estudo buscou avaliar quais plantas medicinais o grupo utilizava no tratamento da doença. Foram encontrados 14 tipos de plantas utilizadas como alternativa terapêutica, sendo o uso do chá das folhas da Bauhinia forficata mais predominante, quando comparado com as outras plantas. $\mathrm{O}$ autor concluiu que $\mathrm{O}$ uso desta planta se torna uma alternativa eficaz para auxiliar no tratamento do DM tipo 2. Portanto sugere-se futuras pesquisas com fármacos associados a Bauhinia forficata, a fim de buscar novas alternativas terapêuticas para auxiliar o tratamento em pacientes com diabetes.

\section{Pterocarpus marsupium roxburgh}

Pterocarpus marsupium roxburgh (PM) também conhecida popularmente como árvore Kino Indiana, pertencente à Família das Fabaceae do gênero Pterocarpus está presente em uma altitude de 200 a $500 \mathrm{~m}$ e seu tamanho varia de moderado a grande podendo chegar até $30 \mathrm{~m}$, com galhos espalhados a PM acaba produzindo um tronco limpo e reto, sua casca tem uma espessura de $1,25 \mathrm{~cm}$, proporcionando um grande número de amostra para fármacos terapêuticos, é nativa da Índia, Nepal e Sri Lanka, fazendo parte de um grande número de plantas que são utilizadas na medicina local, especialmente sua madeira e casca (SUKHADIYA M, et al., 2019; DHAYANEY V e SIBI G, 2019).

Pant DR, et al. (2017), mostrou uma vasta composição fotoquímica com a presença de alcalóides, taninos, terpenóides, carboidratos, flavonóides, glicosídeos, glicosídeos cardíacos, proteína e fenol. Nesse mesmo estudo também foi observado que o extrato etanólico de PM causou um efeito reducional da glicose sanguínea na taxa de $51,30 \%$ na concentração de $200 \mathrm{mg} / \mathrm{kg}$ do extrato etanólico de PM e 55,13\% na concentração de $400 \mathrm{mg} / \mathrm{kg}$, portanto, possuindo ação para o tratamento da diabetes.

Um estudo publicado pelo Journal of Pharmacy Research mostra um comparativo entre o efeito do extrato da casca de PM, da pregabalina e insulina na neuropatia diabética, onde foi analisado diversos fatores como efeito no peso corporal e efeito na glicemia em estado de jejum, para comprovação dos efeitos os camundongos foram induzidos a diabetes do tipo 2. Os resultados desta pesquisa apontaram redução no 
índice glicêmico nos camundongos havendo redução de glicose em jejum inicial de $251,17 \pm 15.30 \mathrm{mg} / \mathrm{dL}$ para $129 \pm 6,26 \mathrm{mg} / \mathrm{dL}$ na concentração de $200 \mathrm{mg} / \mathrm{kg}$ do extrato de $P M$, é de $255,5 \pm 19.62 \mathrm{mg} / \mathrm{dL}$ para $136,67 \pm 8.2 \mathrm{mg} / \mathrm{dL}$ na concentração de $100 \mathrm{mg} / \mathrm{kg}$ do extrato de PM quando aplicado por 8 semanas (GUNASEKARAN V, et al., 2017).

Os efeitos reducionais foram comparados com camundongos tratados com insulina, que demonstrou redução de $252 \pm 12.25 \mathrm{mg} / \mathrm{dL}$ para $147.5 \pm 12.05 \mathrm{mg} / \mathrm{dL}$, o que comprovou que a PM é capaz de reduzir o índice glicêmico no sangue. O extrato atuou no parâmetro glicídico e auxiliou na redução do peso corporal (GUNASEKARAN V, et al., 2017). Assim, futuras pesquisas com fármacos associados a Pterocarpus marsupium roxburgh, podem garantir resultados satisfatórios para diminuição de distúrbio com efeitos colaterais brandos em pacientes com diabetes.

\section{Morus nigra $L$.}

A Morus nigra L, também conhecida como amoreira-preta, amoras-da-horta e amora miúra, pertencente à família Moraceae, é uma planta frútice que chega a atingir entre 3 a 12 metros de altura, que apresenta folhas em formatos diversos como ovóide e picotadas nas bordas, além de apresentar forte e persistente aroma, sendo desenvolvida em regiões de clima tropical e subtropical, na América Central e do Continente Asiático. Amplamente utilizada na produção de seda, no consumo de chás, infusões, na indústria alimentícia, de bebidas e culinária. Seu valor medicinal é devido a sua composição química composta por flavonoides, alcaloides e nutrientes como ácidos orgânicos, vitaminas, glicopeptídeos, bem como compostos que apresentam substâncias responsáveis por efeitos farmacológicos (OLIVEIRA TNFL, et al., 2018).

Araújo CM, et al. (2015), buscou avaliar a eficácia do extrato das folhas e da polpa da planta em um modelo experimental de diabetes. Em um estudo in vivo, observou-se o potencial hipoglicemiante do extrato das folhas de amoreira-preta. Para a realização dos ensaios in vivo, 32 ratas fischer, fêmeas, albinas foram divididas em quatro grupos experimentais: a) controle; b) diabético; c) diabético tratado com polpa; d) diabético tratado com extrato das folhas da amoreira-preta.

Os animais receberam a diabetes por uma injeção intraperitoneal de aloxano, utilizando-se a quantidade de $135 \mathrm{mg} / \mathrm{kg}$. Em um período de 30 dias as ratas foram administradas com o extrato das folhas e a polpa de Morus nigra L. Após a conclusão do tratamento as ratas foram coletadas amostras de fígado e de sangue para as análises bioquímicas e metabólicas. A avaliação dos resultados apresentou que quando as ratas com diabetes foram tratadas com o extrato das folhas de amoreira-preta, houve redução do índice hiperglicêmico quando induzida pelo diabetes, além de elevar as quantidades séricas de insulina, o mesmo efeito não se pode observar com tratamento com a polpa de amora (ARAUJO CM, et al., 2015).

Os resultados apresentaram o extrato das folhas da amoreira-preta como eficazes e eficientes no controle glicêmico em comparação a polpa de amora em modelo experimental de diabetes. Além disso, diversos estudos sugerem não somente atividade antidiabética presente na amoreira-preta, como também outras funções farmacológicas como atividades anti-inflamatórias, antioxidantes, laxante, antimicrobiana, diurética, hepatoprotetora, anti-hipertensiva, analgésica, além de mecanismos que auxiliam o tratamento de indivíduos com alta concentração de colesterol no organismo (TIAN S, et al., 2016).

Segundo Andrade JF e Melnik T (2018), a amoreira-preta apresenta atividade antidiabética presente em seus constituintes, como folhas, frutos, raízes e extratos, tal atividade se dar mediante propriedades iminoaçúcares, como o 1-desoxinojirimicina, um inibidor natural das glicosidases, enzimas que têm a função de hidrolisar os oligossacarídeos não absorvível e polissacarídeos em monossacarídeos absorvíveis, sendo um fator influenciador no atraso da digestão de sacarose, amido, maltose e entre outros carboidratos, assim reduzindo o aumento do nível de glicose no sangue.

Além disso, estudos também apresentam que amoreira-preta apresenta compostos como os flavonoides, que são capazes de desenvolver efeito protetor sobre as células $\beta$ pancreáticas de ratos e os glicopeptídeos, que apresentam atividade semelhante a insulina empiricamente em ratos diabéticos induzidos, concluindo-se atividade antidiabética (ANDRADE JF e MELNIK T, 2018). 


\section{Momordica charantia L.}

A Momordica charantia L (MC) também conhecida como melão de São Caetano ou melão amargo, pertencente à família Cucurbitaceae, é uma planta anual, herbácea, trepadeira que chega a medir de 5 a 7 metros, apresentando folhas membranáceas, fulvecentes e com flores unissexuais, sendo muito comum em regiões do litoral e interior brasileiro e asiático. A MC é popularmente utilizada através de suas sementes, raízes, folhas e frutos para o consumo de chás, infusões, além da produção de medicamentos tradicionais para tratamento de queimaduras, icterícia, dor nas articulações, e distúrbios digestivos, isso, devido suas atividades biológicas, terapêuticas e propriedades nutricionais, constituídas por compostos fenólicos, como hexano, diclorometano, acetato de etila e flavonoides, contribuindo na redução da glicemia em pacientes afetados pelo diabetes (POLITO L, et al., 2016; BORTOLOTTI M, et al., 2019).

Em estudo realizado por Mahmoud MF, et al. (2017), com ratos da espécie wistar, portadores de diabetes induzida por estreptozotocina, evidenciou as atividades antidiabéticas da MC. A planta causou redução da glicose sérica $(135,99 \pm 6,27$ e $149,79 \pm 1,90$ vs. $253,40 \pm 8,18)$ para profilaxia e tratamento. A ação pode se dar devido a presença de substâncias como a lecitina, que possui atividade semelhante à insulina, diminuindo as concentrações de glicose no sangue, além da supressão do apetite. Ela é provavelmente a principal responsável pelo efeito hipoglicêmico que se desenvolve após a ingestão de MC.

França EL, et al. (2014), avaliou os efeitos das nanopartículas de MC e polietileno que são adsorvidas no sangue de pacientes hiperglicêmicos. As amostras de sangue foram coletadas de acordo com o estado glicêmico: normoglicêmico $(N=56)$ e hiperglicêmico $(N=26)$. Determinou-se os efeitos sobre a viscosidade do sangue total do tratamento com um extrato de MC. A viscosidade do sangue de pacientes hiperglicêmicos é maior que o de pacientes normoglicêmicos.

Observou-se que as nanopartículas de extratos de $M C$ reduziram a viscosidade do sangue em taxas equivalentes em indivíduos normo e hiperglicêmicos. Esses dados sugerem que os efeitos da diabetes sobre a viscosidade do sangue devem ser considerados, além de sugerir redução do teor de glicose no sangue. $O$ uso de um extrato de nanopartículas de MC pode representar uma alternativa para o controle e tratamento de doenças do sangue em pacientes diabéticos e além de ser propriedades antidiabéticas (FRANÇA EL, et al., 2014).

Diante da análise dos estudos das plantas acima citadas, observa-se que o uso das plantas medicinais pode ser uma alternativa terapêutica para o tratamento da diabetes. Com isso, este estudo apresenta diversos elementos e compostos que agem estabelecendo o controle glicêmico presente no sangue. Tal controle se dá sob influência dos mecanismos de ações, características e propriedades medicinais presentes nas plantas, seja nas folhas, fruto, decoto ou caule (DEFANI MA e OLIVEIRA LEN, 2017).

Um exemplo é Bauhinia forficata, que apresenta na composição de seu extrato ação antidiabética, assim como a Pterocarpus marsupium, onde os metabólitos secundários, em especial os flavonóides e fenóis, causam efeito reducional da glicose sanguínea. Já a Morus nigra $L$, possui nas folhas e raízes elementos fenólicos, ácido p-cumárico, que podem estar relacionados a diminuição da glicose sanguínea. E por fim a Momordica Charantia L, que a partir dos seus compostos fenólicos, auxilia no tratamento de pacientes diabéticos por meio de chás e infusões (JACKSON T, et al., 2019; MACEDO WDLR, 2019; OLIVEIRA TNFL, et al., 2018; SUKHADIYA M, et al., 2019; POLITO L, et al., 2016).

\section{CONSIDERAÇÕES FINAIS}

Diante ao levantamento das revisões literárias das espécies de plantas Bauhinia forficata, Pterocarpus marsupium roxburgh, Morus nigra L, Momordica charantia L, pode se observar propriedades constituintes com fins terapêuticos que se mostra como uma alternativa para o tratamento da diabetes. Com isso, este estudo, mostra diversos elementos e compostos que agem estabelecendo o controle glicêmico presente no sangue. Tal controle se dá mediante as características e propriedades medicinais presentes nessas espécies que podem estar presentes nas folhas, frutos, caule e raízes. Os estudos aqui demonstrados apresentam potencial como antidiabéticos, podendo auxiliar no tratamento de pacientes acometidos com a doença. A partir do demonstrado entende-se que os resultados apresentados nas pesquisas sobre utilização de plantas e os metabólitos presentes estimulam estudos de novos medicamentos fitoterápicos. 


\section{REFERÊNCIAS}

1. ANDRADE JF, MELNIK T. Efetividade da amora para o tratamento do diabetes mellitus tipo 2: revisão sistemática de ensaios clínicos randomizados. Repositório Institucional UNIFES, 2018.

2. ARAUJO CM, et al. Análise da eficácia da polpa do fruto e do extrato das folhas de amoreira (Morus nigra I.) sobre a modulação de marcadores metabólicos e marcadores do estado redox celular em um modelo experimental de diabetes tipo 1. Instituto de Ciências Exatas e Biológicas, Universidade Federal de Ouro Preto (UFOP), 2015.

3. BORTOLOTTI M, et al. Momordica charantia, a nutraceutical approach for inflammatory related diseases. Frontiers in pharmacology, 2019; 10: 486.

4. COSTA AF, et al. Carga do diabetes mellitus tipo 2 no Brasil. Cadernos de Saúde Pública, 2017; 33 : e00197915.

5. DEFANI MA, et al. Utilização das Plantas Medicinais por Diabéticos do Município de Colorado-PR. Saúde e Pesquisa, 2015; 8(3): 413-421.

6. DHAYANEY V e SIBI G. Pterocarpus Marsupium for the Treatment of Diabetes and Other Disorders. J Complement Med Alt Healthcare, 2019; v. 9(1): 555754.

7. FARIA HTG, et al. Qualidade de vida de pacientes com diabetes mellitus antes e após participação em programa educativo. Revista da Escola de Enfermagem da USP, 2013; 47: 348-354.

8. FRANÇA EL, et al. Effects of Momordica charantia L. on the blood rheological properties in diabetic patients. BioMed research international. 2014: 01-08.

9. GOMES HG, et al. Níveis de conhecimento de pacientes diabéticos sobre a Diabetes Mellitus tipo II. Revista Interdisciplinar, 2018; 11(3): 14-21.

10. GUNASEKARAN V, et al. Neuroprotective role of Pterocarpus marsupium Roxb in streptozotocin-induced diabetic neuropathic pain in Type 2 diabetic rats. Journal Of Pharmacy Research, J Pharm Res, 2017; 11: 1-7.

11. International Diabetes Federation. IDF DIABETES ATLAS. Disponível em: https://www.diabetesatlas.org/en/sections/worldwide-toll-of-diabetes.htmlv. Acesso em: 8 set. 2021.

12. JACKSON T, et al. Biotecnologia Associada ao Monitoramento e Tratamento da Diabetes. Computação \& Sociedade, 2019; 1(1): 152-166.

13. MACEDO WDLR, et al. Uso da fitoterapia no tratamento de doenças crônicas não transmissíveis: revisão integrativa. Revista Brasileira Interdisciplinar de Saúde, 2019; 1(3): 36-43.

14. MAHMOUD MF, et al. Studies on the antidiabetic activities of Momordica charantia fruit juice in streptozotocin-induced diabetic rats. Pharmaceutical biology, 2017; 55(1): 758-765.

15. NOVAES VLSS, et al. CLASSIFICAÇÕES DO DIABETES MELLITUS. Revista Multidisciplinar da Saúde, 2014; 5(10): 19-41.

16. OLIVEIRA JEP, et al. Diretrizes da Sociedade Brasileira de Diabetes 2017-2018. Editora Clannad. 2018. v. 91.

17. OLIVEIRA TNFL, et al. Morus nigra L.: revisão sistematizada das propriedades botânicas, fitoquímicas e farmacológicas. Arch Health Invest. 2018; 7(10): 450-454.

18. PANT DR, et al. Phytochemical screening and study of antioxidant, antimicrobial, antidiabetic, anti-inflammatory and analgesic activities of extracts from stem wood of Pterocarpus marsupium Roxburgh. Journal Of Intercultural Ethnopharmacology. Nepal, 2017; 6(2): 170.

19. POLITO L, et al. A. Plants producing ribosome-inactivating proteins in traditional medicine. Molecules, 2016; 21(11): 1560.

20. SUKHADIYA M, et al. Indian kino tree (pterocarpus marsupium roxb.): biography of excellent timber tree specieS. MFP NEWS, 2019; 29(1): 4-8.

21. TIAN S, et al. Current anti-diabetes mechanisms and clinical trials using Morus alba L. Journal of Traditional Chinese Medical Sciences, 2016; 3: 3-8.

22. TONELLI CA. Avaliação da eficácia clínica de cápsulas contendo extrato padronizado de Bauhinia forficata (pata-devaca) em pacientes diabéticos. 2019.Tese (Doutorado em Ciências da Saúde) - Universidade do Extremo Sul Catarinense, Criciúma. 2019. 